\title{
Obesity and eating disorders: A developmental perspective
}

\author{
LEANN LIPPS BIRCH \\ University of Illinois at Urbana-Champaign, Urbana, Illinois
}

\begin{abstract}
The development of the controls of food intake during the early years of life is discussed, with particular emphasis on the possible role of early experience in the development of eating disorders and obesity. During the first years of life, the change from suckling to feeding occurs. The infant's successful transition from reliance on a single food to consuming a varied diet is essential to adequate growth and health. Infants are probably the only depletion-driven human eaters. By the end of the preschool period, eating occurs as a result of a complex interaction of social, cultural, and environmental factors with physiological cues. Learning and early experience of food and eating play a central role in the development of food-acceptance patterns. During the early years, children are introduced to the diet of their culture and acquire food preferences and aversions, and they learn rules of cuisine, such as when to eat, and even how much to eat. A major theme of this review is that learning, and especially associative conditioning to the social contexts and the physiological consequences of eating, makes major contributions to the formation of food-acceptance patterns during early childhood. This view implicates early experience of eating in the development of individual differences in styles of intake control and could be heuristically useful in the investigation of the etiology of eating disorders and obesity.
\end{abstract}

For the newborn human infant, the feeding situation involves little choice: For young mammals, milk is the food, and infants may be the only depletion-driven human eaters; they eat when they are hungry. Yet although the infant can thrive on a single food, as adult omnivores we need variety in the diet, and this variety is necessary for adequate nutrition by early childhood. This implies that dramatic developmental change occurs in human eating behavior during the early years of life. Cross-cultural evidence also suggests a very high degree of plasticity in the human feeding system; despite the fact that we all begin life on the same diet, across cultural groups there is relatively little overlap in what is consumed. For the adult in our society, eating is rarely depletion-driven, and it tends to occur in response to a complex interaction of physiological feedback with sociocultural factors, including cuisine rules, beliefs about nutrition and health, and environmental cues. A bit of participant observation conducted in the lobby of the convention hotel will reveal adults deciding when, what, where, and with whom to eat. These choices have little or nothing to do with physiological feedback signaling depletion.

The control of food intake in adults is complex, and it is problematic for a large proportion of adults, who have difficulty in maintaining energy balance. Overnutrition is the most widespread nutritional problem in this country today: approximately 34 million adults are obese, and the incidence of childhood obesity is increasing. Chronic

Correspondence should be addressed to Leann Lipps Birch, Child Development Laboratory, University of Illinois at Urbana-Champaign, Urbana, IL 61801. dieting has become normative, especially among women, and the reported incidence of eating disorders is on the rise. Yet problems of overnutrition are not limited to disorders of energy balance. Evidence increasingly implicates diet and hence food selection in the etiology of obesity and chronic degenerative disease: coronary heart disease, atherosclerosis, hypertension, diabetes, and cancer. Current dietary guidelines suggest several dietary changes that need to be made to reduce the incidence of obesity and chronic disease, including reduction of the intake of dietary fat, refined carbohydrate (sugar), and salt (National Research Council [U.S.] Committee on Diet and Health, 1989; U.S. Department of Agriculture, U.S. Department of Health \& Human Services). What follows is a review of findings concerning the contributions made by learning and experience to the development of food preferences and the control of food intake. These findings suggest some reasons why recommended dietary changes can be unappealing, difficult to make, and hard to maintain.

There is little direct evidence on the contributions made by learning and early experience to the pervasive problems of obesity and eating disorders, but existing data suggest some possibilities. First, evidence that infants and young children are responsive to internal cues in the control of food intake will be reviewed, followed by research revealing how learning and socialization in the feeding context can modify the control of food intake. For example, child feeding practices can serve to focus the child on cues other than internal signals of hunger and satiety as controls of food intake. These feeding practices may form the basis for the development of individual differences in styles of intake control that exist among adults. Some of 
the problems of energy balance seen in adulthood may result from styles of intake control in which hunger and satiety cues are not particularly central.

The nature of feeding and the factors that control feeding change dramatically during the first years of life. The infant eats in response to physiological feedback that signals depletion. Milk is the only source of nutrients during the first months of life, but as young omnivores, children require dietary variety to sustain growth and health. Children must make the transition from suckling to being able to accept a variety of foods; and to make this transition, one must learn sociocultural rules and constraints on eating. For example, children learn what constitutes food in their culture, as well as what is disgusting. Food likes and dislikes are shaped by learning and experience. Children are very neophobic, but with repeated exposure, they come to accept foods (Birch \& Marlin, 1982). They also learn when to eat (Birch, McPhee, Sullivan, \& Johnson, 1989), which foods are eaten at particular mealtimes (Birch, Billman, \& Richards, 1984), and what foods and flavors can be appropriately combined. Learning also influences how much children eat of familiar foods.

Children acquire many of the basic rules of cuisine by the time they are 5 or 6 years old, at which time the depletion-driven eating of infancy has been transformed; at that point in development, eating is controlled by a complex interaction of depletion cues with sociocultural rules and environmental cues. Learning occurs as a result of the redundant nature of eating, which affords many opportunities for associative conditioning. Patterns of intake come increasingly under the control of sociocultural and environmental cues; individual differences in patterns of intake control emerge during childhood and adolescence, on the basis of differences in experiential history. By the time of adulthood, there exist individual differences in styles of intake control that have been well documented, although views differ on the dimensions of these differences. For example, some adults are very responsive to physiological cues of hunger and satiety, but others are chronic dieters who use a variety of cognitive strategies to control food intake.

\section{RESPONSIVENESS TO ENERGY DENSITY: THE CONTROL OF FOOD INTAKE IN EARLY CHILDHOOD}

Six-week-old infants adjust their intake in response to the energy density of formula, consuming larger volumes of dilute than of concentrated formula (Fomon, 1974). Evidence from our laboratory reveals that preschoolers also adjust intake in response to the energy density of foods. In this research, children consumed meals or snacks that consisted of two courses: fixed volumes of yogurts, drinks, puddings, or ice creams that were either high $(150 \mathrm{kcal} / 100 \mathrm{ml})$ or low $(50 \mathrm{kcal} / 100 \mathrm{ml})$ in energy density, followed by an ad-lib course consisting of an array of palatable foods. The results of such single-meal protocols revealed that children are responsive to the energy density of the preload, eating more ad lib following the low- as opposed to the high-density preload (Birch \& Deysher, 1985, 1986; Birch, McPhee, \& Sullivan, 1989). The results of one such experiment appear in Figure 1. In one study, in which children's intake in this type of protocol was compared with that of adults, the children showed clear evidence of responsiveness to energy density, whereas the adults did not. How do children alter their intake patterns to produce these adjustments in energy intake? The results of one series of experiments indicated that following a high-energy-density preload, children consumed a more restricted range of foods than they did following the low-energy-density preloads, and they restricted their consumption to their more preferred foods (Birch, McPhee, \& Sullivan, 1989).

The nutritive consequences of foods can also serve as unconditioned stimuli in the conditioning of preferences and conditioned satiety. Following the work of Sclafani (1990), we have shown that when children have repeated experience of eating two foods-a high-energy-density version and a low-energy-density "diet" version, they learn to prefer the flavor associated with high energy density (Birch, McPhee, Steinberg, \& Sullivan, 1990). To the extent that such conditioning occurs as a result of our everyday eating experiences, this learning could be contributing to our preferences for energy-dense foods. Current dietary guidelines recommend cutting down on energy-dense foods in order to improve the quality of the diet and reduce the chances of obesity and chronic disease. If we have learned to prefer such foods, it helps to explain why it is so difficult to comply with these dietary guidelines.

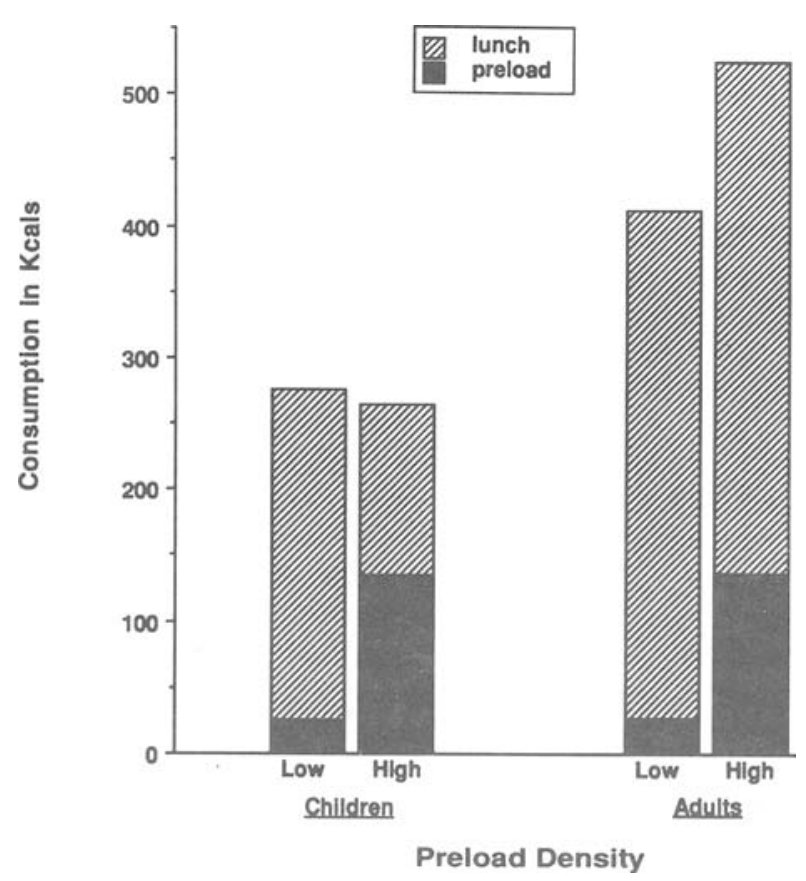

Figure 1. Children's and adults' energy intake at lunch, including high- and low-energy-density preloads. 
Children can also use energy density as an unconditioned stimulus in the conditioning of satiety (Birch \& Deysher, 1985; Birch, McPhee, Shoba, Steinberg, \& Krehbiel, 1987). In this case, the amount of a food consumed is adjusted over time in response to its energy density. During conditioning trials, children are given repeated experience in consuming fixed volumes of two distinctively flavored versions of a food, one high and one low in energy density. Following this, the children eat a self-selected meal from an array of palatable foods, and their ad-lib consumption provides the data of interest. With repeated experience, children can learn to associate the flavor cues with the caloric consequences of the foods and to anticipate how filling and how fatty a particular food is and adjust intake accordingly. This is revealed in extinction test trials in which the energy density difference between the foods is removed, and the flavors are presented in isocaloric preparations. As is shown in Figure 2, children continue to eat less ad lib following the flavor that has previously been paired with high energy density.

The evidence presented above is restricted to single meals. What about the control of intake over a longer period of time? One of the most common observations regarding children's food intake is that their eating at individual meals is erratic and very unpredictable; a child will eat "like a bird" on one occasion and "like a horse" on the next. Clara Davis's descriptions of the children's food intake in her dietary self-selection studies conducted 60 years ago paint a similar picture (Davis, 1928, 1939). Despite this, she reported that the children grew well and were healthy, regardless of the absence of adult attempts to control their food intake. Recent evidence suggests that despite the erratic nature of intake at individual meals, total daily energy intake is relatively tightly regulated by young children (Birch, Johnson, Andresen, Peters, \& Schulte, 1991).

To investigate the control of energy intake by young children, we obtained 24-h food intake data from 152 to 5-year-old children for 6 days. Coefficients of variation (CV) were calculated for each child for each of the six eating occasions per day (breakfast, lunch, dinner, and three snacks) and for total daily energy intake. The coefficient of variation is the standard deviation divided by the mean, making it possible to compare the variability of measures with very different mean values, as in comparing the variability of energy intake at individual meals with the variability of energy intake over $24-h$ periods. These CVs for individual children are presented in Figure 3, which reveals that the children's intake at individual meals was highly variable, but that total energy intake was relatively tightly regulated: The mean within-subjects $\mathrm{CV}$ for energy intake at individual meals was $33.6 \%$; in contrast, the mean within-subjects coefficient of variation for total daily energy intake was $10.4 \%$. Evidence that compensation in intake across successive meals produced the relative consistency in total daily intake was revealed by a pattern of predominantly negative correlations between intake at successive pairs of meals. When we examined correlations between energy intake at one meal and the next, a preponderance of these correlations were negative, providing evidence that compensation across suc-

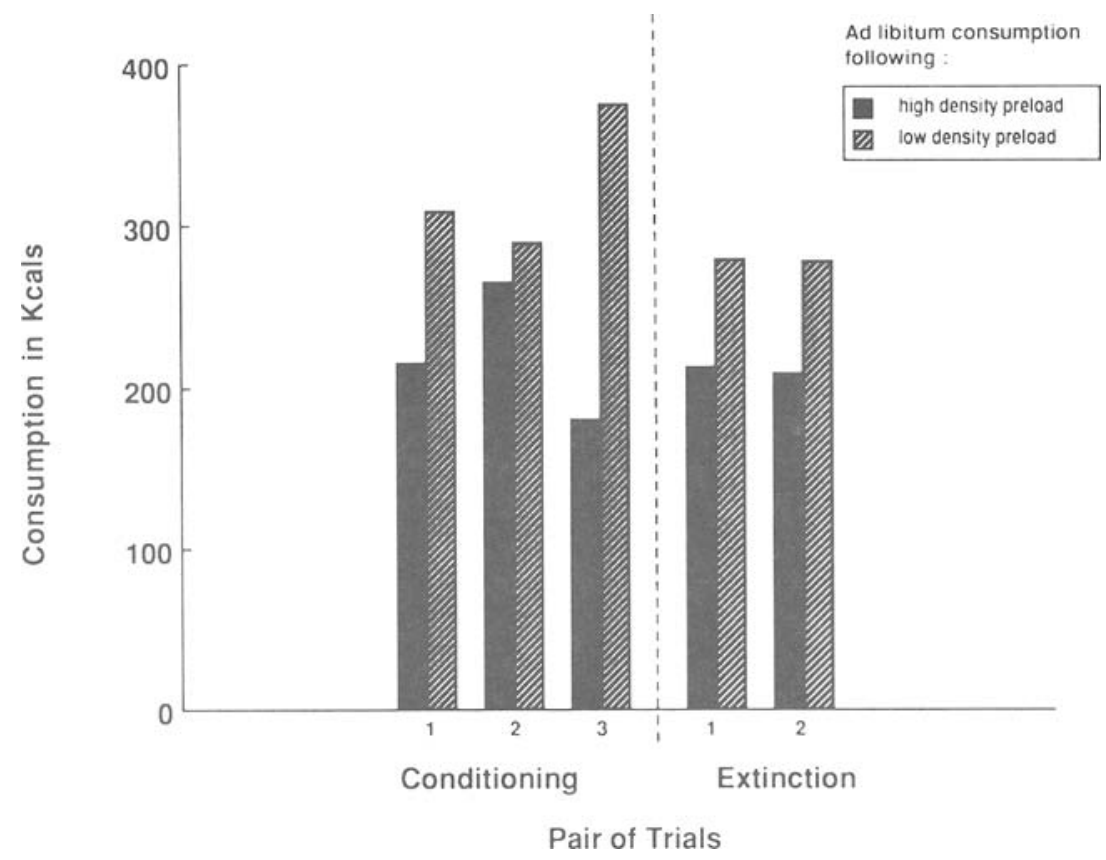

Figure 2. Ad-lib energy intake following high- or low-energy-preload paired flavors during conditioning and extinction: Evidence for conditioned satiety. During extinction, preparations previously high or low in energy density are isocaloric. 


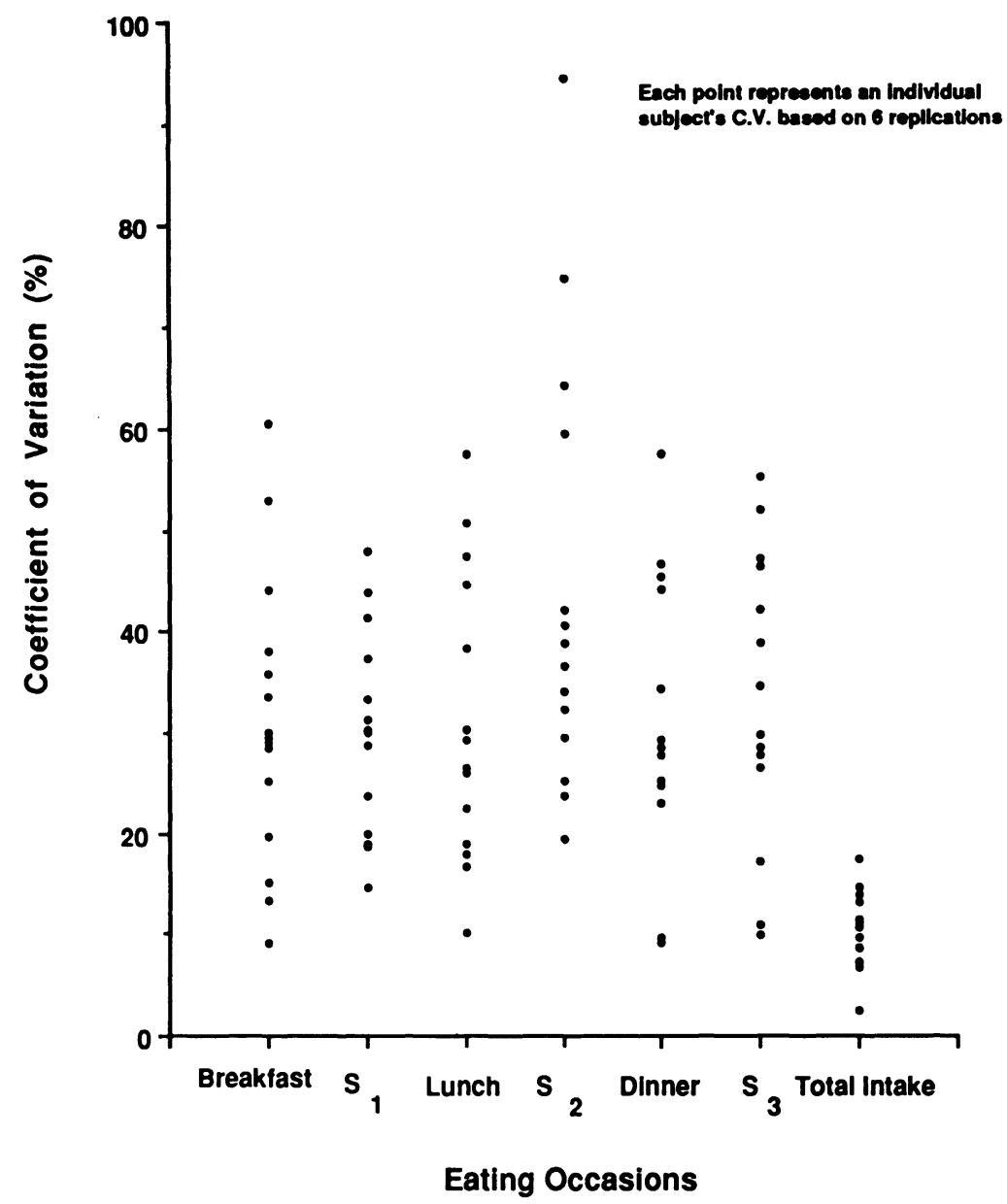

Figure 3. Coefficients of variation for energy intake of 15 preschool children at each of six meals and for total daily energy intake.

cessive meals produced the relative consistency of total daily energy intake. Examination of the relationship between individual subjects' CVs for total energy and mealto-meal compensation provided further evidence that compensation in energy intake across successive meals was producing this relative consistency of total daily energy intake; children with the lowest CVs for total daily energy intake showed the strongest evidence of meal-to-meal compensation in energy intake $(r=-.51, p<.05)$.

To summarize, evidence both from single-meal protocols and from studies of total daily energy intake indicates that children are sensitive to the energy density of foods and use such nutritive feedback in controlling their food intake. All this corroborates what Davis said 60 years ago: In the absence of adult intervention, and given nutritious choices, children can select an adequate diet (1928). Notice that this statement is qualified by "in the absence of adult intervention." This is an important point that I will return to, because young children's eating seldom occurs in the absence of adult intervention.

\section{THE SOCIAL CONTEXT OF FEEDING AND THE DEVELOPMENT OF FOOD-ACCEPTANCE PATTERNS}

\section{Learned Preferences and}

\section{the Acquisition of Cuisine Rules}

During the preschool years, children are gradually introduced to many of the foods of the adult diet of their culture. The quality and quantity of early experience with foods is pivotal in determining food-acceptance patterns. The redundant nature of eating provides many opportunities for associative conditioning involving the contexts and consequences of eating. In this section, evidence is presented which indicates that the social context of feeding contributes substantially to food-acceptance patterns. Through repeated experience with food, children learn preferences and aversions, cuisine rules about appropriate food-flavor combinations, and times of day at which foods are consumed. Child feeding practices also provide children with information regarding which of the many 
cues available should control the initiation, maintenance, and termination of eating.

Why is it that children seem to dislike foods that are good for them and love the things that are bad for them? We have evidence that child feeding practices used by adults to ensure that the child obtains a nutritious diet actually contribute to this state of affairs. Child feeding practices often involve cajoling, wheedling, or coercing children to eat, and these are often used when the parent believes that the child cannot adequately control intake, or possibly that the child is at risk for problems of energy balance. We have simulated some of these child feeding practices in the laboratory in order to look at their effects on food preferences. Results of our research reveal rather paradoxical results: children learn to dislike foods that parents reward them for eating (Birch, Marlin, \& Rotter, 1984). Conversely, foods parents use as rewards are often high in fat, sugar, or salt, and these foods become even more preferred as a result (Birch, Zimmerman, \& Hind, 1980).

Children also seem to be learning about whether or not it is appropriate to add sugar or salt to foods, and these cuisine rules seem to be extracted from children's experience in eating foods either with or without added sugar or salt (Sullivan \& Birch, 1990). We gave children repeated experiences of eating a new food (tofu) that was prepared one of three ways: plain, or with sugar or salt added. As in previous experiments, the children were initially quite neophobic, and they did not readily eat the new foods. However, with repeated exposure, the children came to accept and eat whatever version they had experienced. However, following their experience with one version, they actually increased their dislike for the versions they had not eaten relative to their initial dislike, suggesting that the children were not just learning to like one food/flavor combination, but were learning to dislike the "inappropriate" version. This pattern of increased liking for the familiarized version, along with increased dislike for the other version, was noted regardless of whether the familiarized food was flavored or plain. The finding that children are learning appropriate food contexts for the addition of sugar and salt suggests that if we want children to accept foods that are lower in sugar and salt, foods for them should be consistently prepared with low levels of sugar and salt. Although there is little systematic research on the sugar and salt content of young children's diets, the results of one study in which children's food intake was monitored provide evidence that foods most frequently consumed by children are very high in sugar, salt, and fat (Davidson, Hayek, \& Altschul, 1986).

We recently conducted research to determine whether environmental cues that had previously been paired with eating could lead to the initiation of eating in children who were not hungry. Such evidence would provide support for the view that by early childhood, eating is no longer depletion-driven, but can be controlled by a variety of other cues. This research was inspired by that of Weingarten (1983), who was able to demonstrate conditioned initiation of eating in sated rats. In our case, children participated in free play sessions in two different playrooms on different days. In one playroom, food was always present; in the other, food was never present. After the children had acquired repeated experience of these two settings, we tested them for conditioning effects. On test trial days, the children were fed a large dish of Haagen-Dazs ice cream $(260 \mathrm{kcal})$ to ensure that they were not hungry, and then they were sent to the playroom for free play. On the test trials, food was present in both settings. Latency to eating and ad-lib consumption in the two contexts are presented in Figure 4, which provides evidence for conditioned meal initiation. Children can learn to initi-

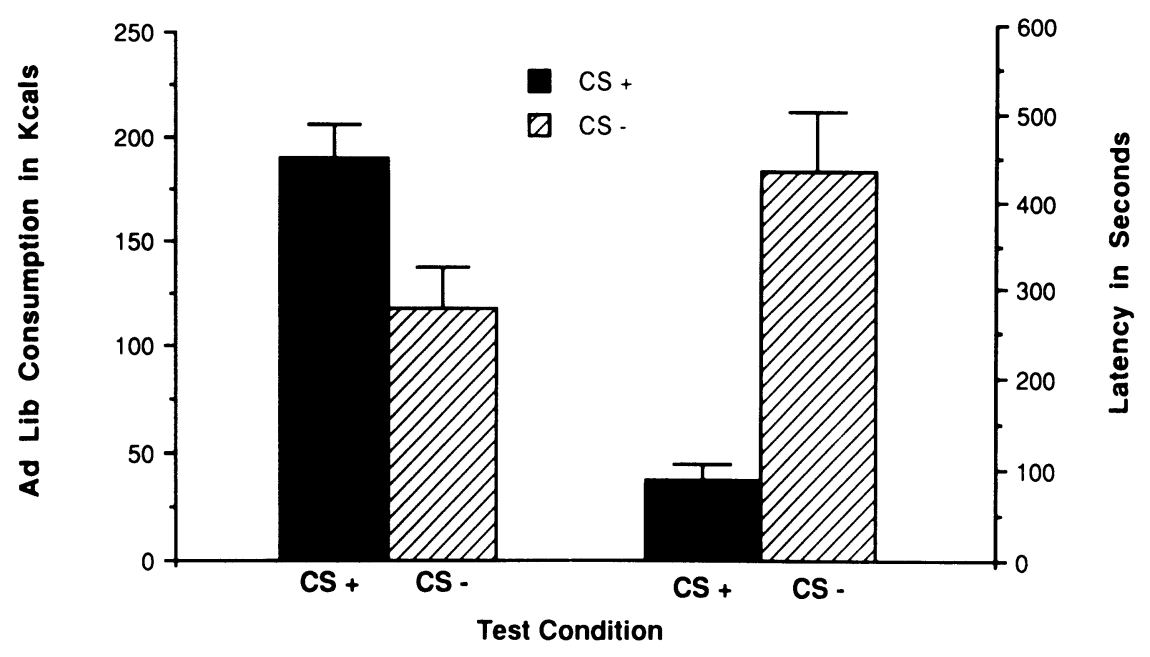

Figure 4. Conditioned meal initiation in children. Ad-lib consumption and latency to eating in contexts where food had (CS+) or had not (CS-) previously been available. 


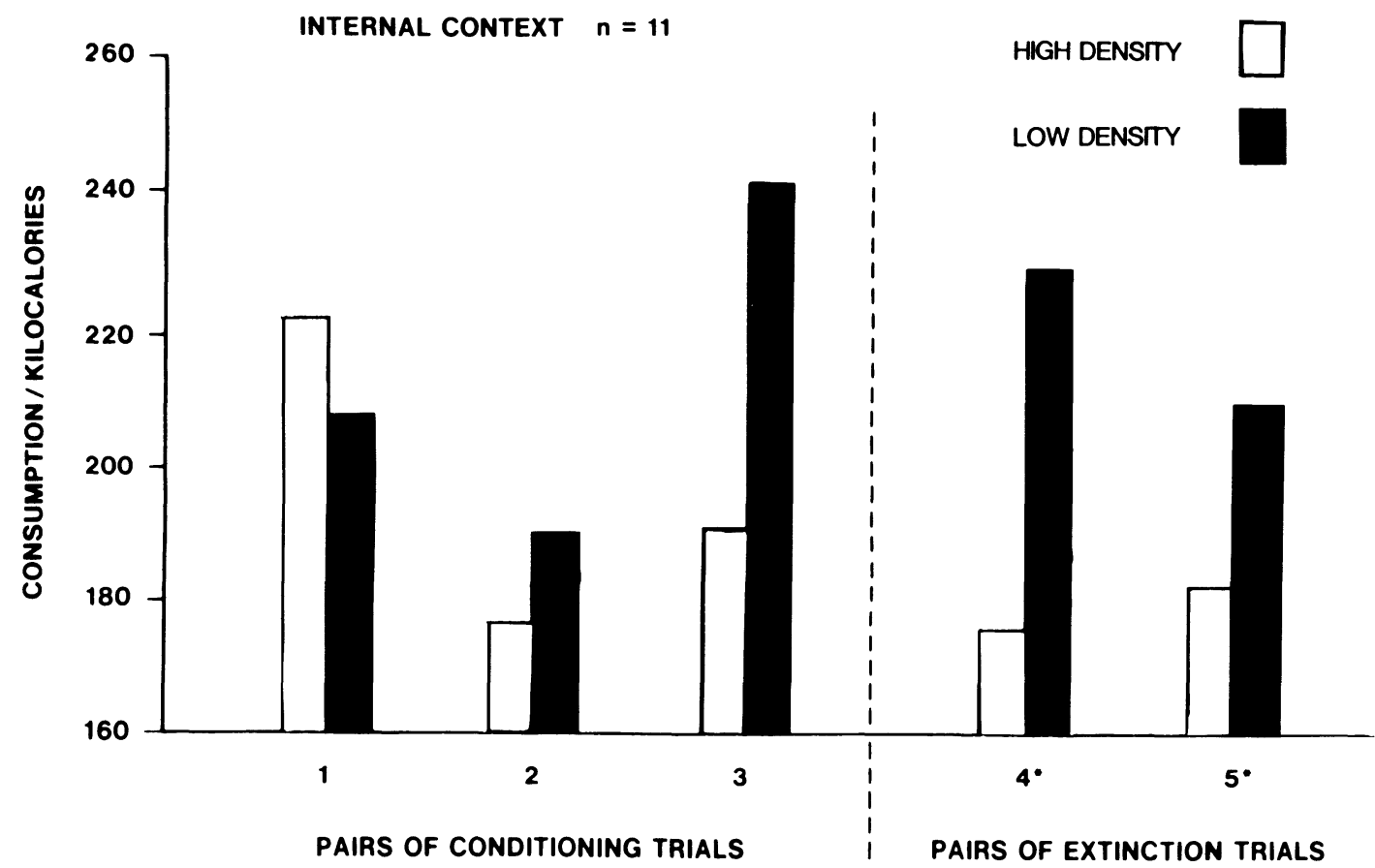

EXTERNAL CONTEXT $n=11$

HIGH DENSITY

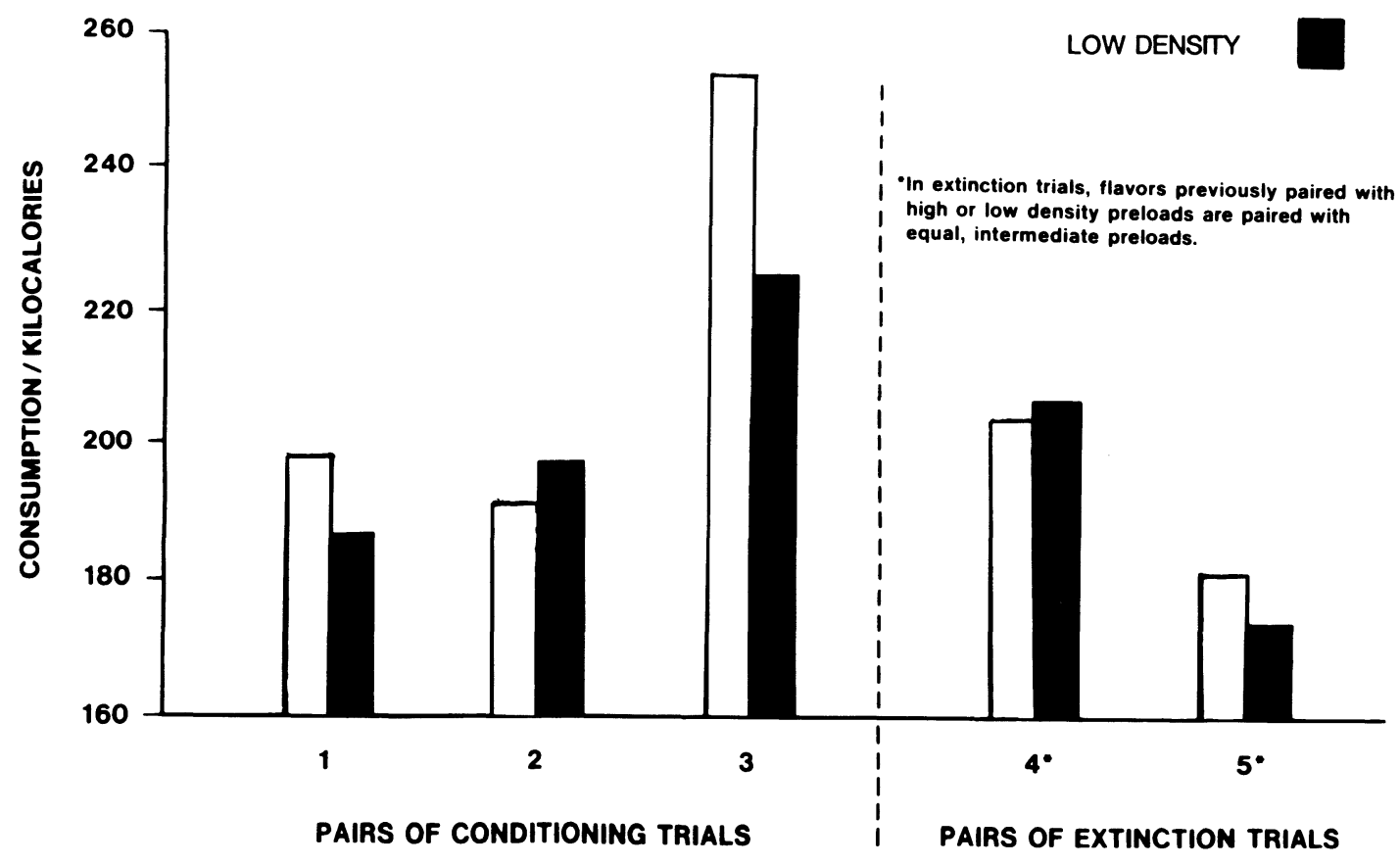

Figure 5. Social context of feeding modifies responsiveness to energy density and the conditioning of satiety: Ad-lib energy intake following high- and low-energy-density paired flavors. The "internal" social context focused children on hunger and satiety as controls of food intake; the external context focused on external cues controlling intake. 
ate meals in response to contextual cues, and to eat in the absence of depletion cues, providing a striking contrast to the depletion-driven eating of infancy.

\section{The Social Context of Feeding and the Etiology of Individual Differences in Intake Control}

In addition to influencing the formation of specific food preferences, there is evidence that child feeding practices that involve a high degree of external control of the child's eating may have the effect of teaching the child which of the many cues available to control feeding should be attended to and used. For example, if the child says "I'm full" and the parent says, "Finish what's on your plate," or if the child says "I'm hungry" and the parent says "It's not time to eat now," the parent is sending a clear message that internal cues signaling hunger and satiety should be ignored in favor of external cues, such as time of day and amount of food remaining on the plate. In the absence of adult controls, children are responsive to energy density in controlling their food intake (Birch \& Deysher, 1986; Birch, McPhee, \& Sullivan, 1989). We also have experimental evidence that child feeding practices can modify the extent to which children are responsive to internal cues (Birch et al., 1987). In one experiment, we replicated the research on the conditioning of satiety in two different child feeding contexts. In the "internal" context, the adults feeding the children talked about feelings of hunger and satiety, and how they could tell when to eat and when to stop eating. The children also had the chance to feed a doll with a clear glass stomach, which provided another context for the discussion of hunger and satiety. Again, children received repeated experience with consuming fixed volumes of two distinctively flavored versions of a food, one high and the other low in energy density, followed by ad-lib consumption of a self-selected snack. In the external context, the same procedures were followed, but the adults emphasized external cues for eating: A bell rang, signaling time for the snack; the children were focused on the amount of food remaining; and they were given stickers contingent on eating-all of which were procedures designed to simulate common child feeding practices. During extinction test trials, flavors were presented in isocaloric versions of the foods as before, and the internal and external social contexts were eliminated.

The results, shown in Figure 5, revealed evidence of responsiveness to energy density prior to learning and evidence of conditioning only for the internal group. The external group showed evidence of a reinforcement effect, in that the children increased their consumption in response to the contingency, but there was no evidence that responsiveness to energy density controlled their intake during conditioning or extinction. These results suggest that child feeding practices can produce differences in the extent to which children are responsive to internal feedback in controlling their energy intake.

\section{SUMMARY AND CONCLUSIONS}

There is clear evidence that infants and young children are responsive to the energy density of foods in controlling their food intake. Children can use the physiological feedback arising from differences in energy density to learn to anticipate the fillingness or satiety value of a familiar food and adjust intake accordingly. More recent research has revealed that children learn to prefer food flavors that have been repeatedly associated with highenergy-density over "diet" versions of the same foods. While there is evidence that children have mechanisms available for controlling energy intake, there is limited information that the extent to which such mechanisms are used by children can be readily altered, in response to child feeding practices and in response to the context of feeding. Children learn which of the many cues available to control eating should be salient, and I would suggest that individual differences in styles of intake control seen in adulthood may begin to emerge with the imposition of child feeding practices that differ (1) in the extent of external control, and (2) in the array of foods typically made available to children. In addition, there is evidence that child feeding practices influence the formation of food preferences through associative conditioning of food cues to the social context of eating.

\section{REFERENCES}

Birch, L. L., Billman, J., \& Richards, S. (1984). Time of day influences food acceptability. Appetite, 5, 109-112.

BirCH, L. L., \& DEYSHER, M. (1985). Conditioned and unconditioned caloric compensation: Evidence for self regulation of food intake by young children. Learning \& Motivation, 16, 341-355.

BirCH, L. L., \& DeYSher, M. (1986). Caloric compensation and sensory specific satiety: Evidence for self-regulation of food intake by young children. Appetite, 7, 323-331.

Birch, L. L., Johnson, S. L., Andresen, G., Peters, J. C., \& SCHULTE, M. (1991). The variability of young children's energy intake. New England Journal of Medicine, 324, 232-235.

BIRCH, L. L., \& MARLIN, D. W. (1982). I don't like it; I never tried it: Effects of exposure on two-year-old children's food preferences. Appetite, 3, 353-360.

Birch, L. L., MARLin, D., \& Rotrer, J. (1984). Eating as the "mean" activity in a contingency: Effects on young children's food preference. Child Development, 55, 432-439.

Birch, L. L., McPhee, L., Shoba, B. C., Steinberg, L., \& Krehbiel, R. (1987). "Clean up your plate": Effects of child feeding practices on the conditioning of meal size. Learning \& Motivation, 18, 301-317.

Birch, L. L., McPhee, L., Steinberg, L., \& Sullivan, S. (1990). Conditioned flavor preferences in young children. Physiology \& Behavior, 47, 501-505.

Birch, L. L., McPhee, L., \& Sullivan, S. (1989). Children's food intake following drinks sweetened with sucrose or aspartame: Time course effects. Physiology \& Behavior, 45, 387-396.

Birch, L. L., McPhee, L., Suluvan, S., \&ohnson, S. (1989). Conditioned meal initiation in young children. Appetite, 13, 105-113.

BirCH, L. L., Zimmerman, S., \& HiNd, H. (1980). The influence of social affective context on preschool children's food preferences. Child Development, 51, 856-861.

Davidson, F. R., Hayek, L.A., \& Altschul, A. M. (1986). Towards 
accurate assessment of children's food consumption. Ecology of Food \& Nutrition, 18, 309-317.

DAvis, C. (1928). Self selection of diet by newly weaned infants. American Journal of Diseases of Children, 36, 651-679.

DAvIS, C. (1939). Results of the self selection of diets by young children. Canadian Medical Association Journal, 41, 257-261.

Fomon, S. J. (1974). Infant nutrition (2nd ed.). Philadelphia: W. B. Saunders.

National Research Council (U.S.) Committee on Diet and HEALTH (1989). Diet and health: Implications for reducing chronic disease risk. Washington, DC: U.S. Government Printing Office. SCLAFANI, A. (1990). Nutritionally-based learned flavor preferences in rats. In E. D. Capaldi \& T. L. Powley (Eds.), Taste, experience, and feeding (pp. 139-156). American Psychological Association.

Sullivan, S., \& Birch, L. (1990). Pass the sugar, pass the salt: Experience dictates preference. Developmental Psychology, 26, 546-551.

U.S. Department of Agriculture, U.S. Department of Health \& Human Services (1985). Nutrition and your health: Dietary guidelines for Americans (Home and Garden Bulletin No. 232, ed. 2). Washington, DC: U. S. Government Printing Office.

WEINGARTEN, H. (1983). Conditioned cues elicit eating in sated rats: A role for learning in meal initiation. Science, 220, 431-433. 